\title{
Comparison of Pollution Level of Aged Porcelain and Silicon Rubber Insulators
}

\author{
M. A. Salam, S. P. Ang, Fushuan Wen, M. Fadil, H. \\ A. Hadi, \\ Dept. of Electrical and Electronic Engineering \\ Faculty of Engineering, Institut Teknologi Brunei, \\ Bandar Seri Begawan, Brunei Darussalam \\ Email: abdus.salam@itb.edu.bn
}

\author{
Q. M. Rahman, William Voon \\ Dept. of Electrical and Computer Engineering \\ University of Western Ontario, London, Canada \\ Berakas Power Management Company \\ Bandar Seri Begawan, Brunei Darussalam \\ Email: qrahman@eng.uwo.ca
}

\begin{abstract}
In this paper, an artificial neural network (ANN) has been used to compare the pollution level of aging long-rod silicon rubber and porcelain insulators. High regression values and effective correlation of equivalent salt deposit density (ESDD) have been found using MATLAB neural network toolbox. It has been found that the bottom surface of the porcelain insulator contains more pollution than the top surface. Higher pollution has also been measured in case of silicon rubber (SiR) insulator. Pollution levels in case of both insulators have been found to be exceptionally high compared to IEEE and CIGRE standards.
\end{abstract}

Keywords-long-rod insulators; ESDD; determination of coefficient

\section{INTRODUCTION}

Day by day demand in electric power is increasing due to the rapid growth in industrial sectors, commercial buildings and residential areas. To meet this demand, new power stations need to be built and the efficiency of transmission systems needs to be improved. However, maintaining efficiency of a transmission system is a challenging task due to the presence of unpredictable faults. Failure to mitigate these unpredictable faults results in economic losses for companies and consumers. One such challenge is encountered when high voltage insulators cause flashover and discontinuity of power supply on a large scale, after being affected by different pollution sources [1]. In Brunei Darussalam, porcelain, glass and silicon rubber insulators are used in $66 \mathrm{kV}$ transmission lines. These insulators are polluted with sea salt, dust, chemicals, and ashes. The ashes are normally the outcome of bush fire during the months of March and April in each year. Sometimes, there are some power outages in the Brunei transmission networks due to combination of different types of pollutions. Pollution is one of the main problems which affect the efficiency of the insulators. Over the period of time, pollution in the air settles on the surface of the insulators and mixed with dews and rain which offers conductive layer. The leakage current flows in the conductive layer of the insulator and creates uneven surface voltage. This uneven surface voltage finally triggers insulator flashover [2]. In the recent past, the leakage current measurement method has been used to prepare the exact pollution maps and optimal design of insulators for the transmission lines and substations [3]. In Brunei Darussalam, the length of the transmission lines falls into the category of short transmission and medium transmission lines due to the country's small geographical size. The transmission lines pass along the highways near coastal area. There are two types of arrangements installed for the insulators used, double-I-string and V-string [4]. It is important to measure the level of pollution which helps to improve the efficiency of transmission lines. The pollution level in high voltage insulators can be measured by several methods, namely, equivalent salt deposit density (ESDD), non-soluble deposit density (NSDD) and pollution index (PI). In this study, the ESDD has been used to measure the pollution level. An artificial neural network (ANN) has been chosen to analyze the measurement of ESDD due to ANN's popularity in this domain [5].

\section{Measurement Of Pollution LeVEl}

Two heavily polluted, and approximately 30 years old insulators were brought in from a local utility company, for the pollution-measurement experiment. Some parts of those insulators were found to be cracked. However, extreme cautions were taken during collection of the pollutants. The insulators were brought to the laboratory, where, the pollutants were collected by brushing it off, and later on mixed with distilled water for making salt-solution. These two insulators are long-rod porcelain and long-rod silicon rubber ( $\mathrm{SiR}$ ) ones. The long-rod porcelain insulator has 15 layers, ' $a$ ' to 'o', where 'o' has the highest potential and 'a' has the lowest potential. The long rod silicon insulator has 7 layers, ' $a$ ' to ' $g$ ', where ' $a$ ' has the highest potential and ' $g$ ' has the lowest potential.

The equipment YSI Model 63 has been used to measure the solution temperature, $\mathrm{pH}$ and conductivity. These measured conductivities at different temperatures are then converted to the corrected conductivities at $20^{\circ} \mathrm{C}$ by the following formula [6]:

$$
\sigma_{20}=\sigma_{\theta}[1-b(\theta-20)]
$$

In addition, the value of salinity, $S_{a}$ of the solution can be expressed by the following empirical formula:

$$
S_{a}=\left(5.7 \sigma_{20 \mu S / \mathrm{cm}} \times 10^{-4}\right)^{1.03}
$$

Finally, the Equivalent Salt Deposit Density (ESDD) is determined by using the following expression, 


$$
E S D D=\frac{\left(S_{a} \times V\right)}{A}
$$

The pollution levels in terms of ESDD at the bottom and top surfaces of both the long-rod porcelain and silicon rubber insulators have been measured.

\section{ARTIFICIAL NEURAL NETWORK}

A neural network tool has been developed using MATLAB 2014a and represented as ANN model as shown in Fig. 1. In Table 1, four inputs, two hidden layers with five neurons each and an output layer with only one neuron have been considered [6]. The selected inputs are salinity, conductivity, volume of water and surface area of insulators and the output of this model is Equivalent Salt Deposit Density (ESDD).

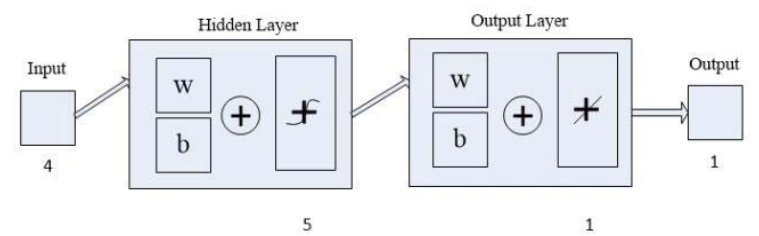

Fig. 1. Neural network modeling.

The input value varies from -1 to 1 and the input layer distributes the values to each of the neuron in the hidden layer. The constant input of this network is called bias whose value is 1.0 and it is contributed to each of the hidden layers. The bias is multiplied by a weight and added to the sum going into the neuron. In hidden layers, the value from each neuron is multiplied by a weight. The weighted values are added together into a transfer function. Then the outputs are distributed into the output layer. Likewise, the output layer has the same calculations with hidden layer but producing different output values. Both ANN values and predicted values for each insulator have been implemented into the network.

In this model, there are three different sets of performing neural networks, training, validation and testing. The training set has been used to adjust weights on the neural network and the validation set has been used to determine the accuracy of the data. If the accuracy of the data stays the same or below the threshold, the neural network is over-fitting and the network should be stopped. Testing set is used for testing the final solution in order to confirm the actual predictive power of the network.

Table I. The characteristics of the ANN model.

\begin{tabular}{|c|c|}
\hline Item & Value \\
\hline Network Type & Feed Forward \\
\hline No. of Layers & 3 \\
\hline Input Layer Nodes & 4 \\
\hline Hidden Layer Nodes & 5 \\
\hline Output Layer Nodes & 1 \\
\hline Transfer Function & LOGSIG \\
\hline
\end{tabular}

There are four basic steps in the training process. These are: assemble the training and predicted data, create different network layers, train the network and simulate the network layers to generate new outputs. Artificial neural network algorithm has been used successfully in many applications. It is important that it acts as a model of real-world system or function. The formulae given in $[5,7,8,9,10]$ can determine the output from its input accordingly. For each neuron in the input layer, the neuron outputs are given by,

$$
O_{i}=\text { net }_{i}
$$

Where $n e t_{i}$ is the input of neuron $i$, and $O_{i}$ is the output of neuron $i$. Also, for each neuron in the output layer, the neuron inputs are given by,

$$
n e t_{k}=\sum_{j=1}^{N_{j}} w_{k j} O_{j}, \quad k=1 \ldots . . N_{k}
$$

Where $w_{k j}$ is connection weight between neuron $j$ and neuron $k$, and $N_{j}, N_{k}$ are number of neurons in the hidden and output layers, respectively. The neuron outputs are given by,

$$
O_{k}=\frac{1}{1+\exp \left(n e t_{k}+\frac{\theta_{k}}{\theta_{o}}\right)}=f_{k}\left(\text { net }_{k}, \theta_{k}, \theta_{o}\right)
$$

Where $\theta_{k}$ is threshold of neuron $k$, and the activation functions $f_{k}$ is a sigmoidal function. For the neurons in the hidden layers, the inputs and the outputs are given by the relationships similar to those given in equations (5) and (6), respectively.

\section{RESUlTS AND Discussions}

In MATLAB, measured data have been implemented into the workspace namely, input, output and predicted data from the Microsoft's Excel workspace, as shown in Fig. 2.

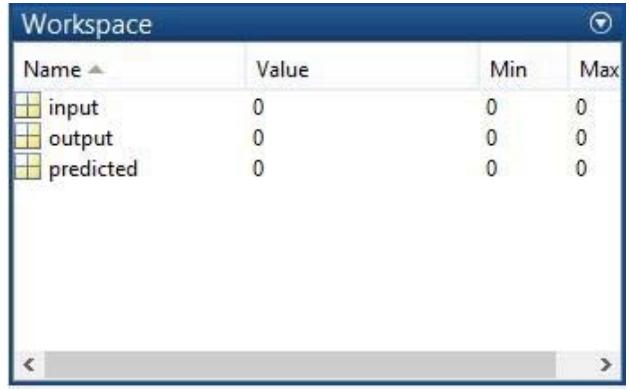

Fig. 2. Workspace on MATLAB window.

The input variables of workspace are inserted by the selected inputs. These input parameters show better correlation with the given formulae to calculate ESDD. The measured input, output and predicted parameters must be in between -1 and 1 because the network has been created based on the sigmoidal neuron model. An artificial neural network's command 'nntool' was used to activate the simulation. The input and predicted data are considered as input variable for getting output as target variable.

The input and output variables are placed into the workspace for evaluating the predicted values by considering an average of each input parameter respectively. All readings 
are normalized before starting the simulation. Different network layers have been created using feed-forward back propagation algorithm. The network type used is feed-forward in which the information is propagated in one direction.

The data are trained depending on how long it takes. When more time is consumed, more accurate the outputs become by inserting a value of 1000 into max_fail in training parameters. The parameter, max_fail represents the maximum number of validations. As the number of training cycle increases, the input-output relationship show better correlation within the same data-range.

In Fig. 3, the data are trained to give new predicted ESDD in terms of training, validation and testing.

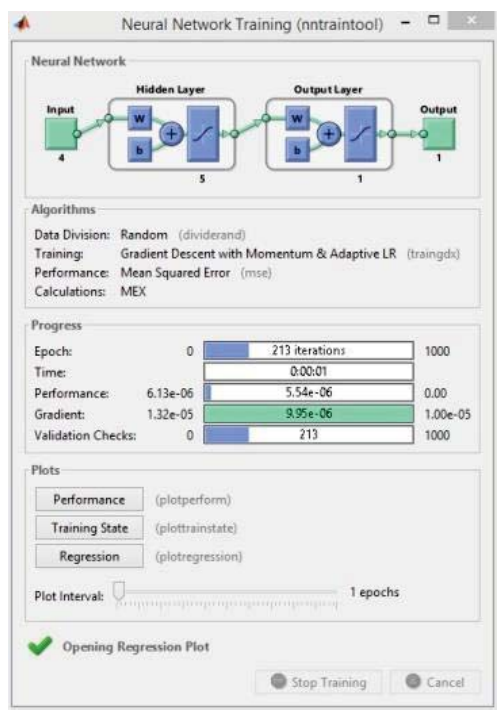

Fig. 3. Neural network training.

The predicted ESDD values are considered as the output, and calculated ESDD values are considered as target. The output for silicon rubber and porcelain insulators are plotted against the target for training, validation and testing as shown in Figs. 4, 5, and 6, respectively. The input parameters have been trained to get the target ESDD (measured ESDD). The input layers from the ANN values of either long-rod silicon rubber insulator or long-rod porcelain insulator are being calculated depending on number of cycles. This gives the results in terms of output for all three stages namely training, validation and testing. The performance of the neural network model is measured by using regression $\mathrm{R}$ value. The term regression $\mathrm{R}$ value is the measurement of the correlation between outputs and targets. A total of 7 discs of long rod silicon rubber insulator have been considered for data collection for both top $(T)$ and bottom $(B)$ surfaces as shown in Table II. Each reading has been used to evaluate a different set of ESDD values. These readings have actual ESDD and predicted ESDD. Unlike the values of actual ESDD which are being calculated, the values of predicted ESDD are evaluated by using the neural network in MATLAB. The comparison between actual ESDD and predicted ESDD shows that the values are more or less the same for each disc respectively.
In case of long rod porcelain insulator, the values of $\mathrm{R}$ are $0.98139,1$ and 1 in training, validation and testing, respectively. The regression line for training, validation and testing stages are determined as shown in Figs. 4, 5, and 6 accordingly. Unlike silicon rubber insulator, a total of 15 discs of porcelain insulator have been considered for data collection for both top $(T)$ and bottom $(B)$ surfaces as shown in Tables III. As the values of corrected ESDD, which are being calculated, the values of predicted ESDD are evaluated by using the neural network in MATLAB too. The ANN is used in this paper to find the better coefficient of determination between the measured and predicted ESDD.

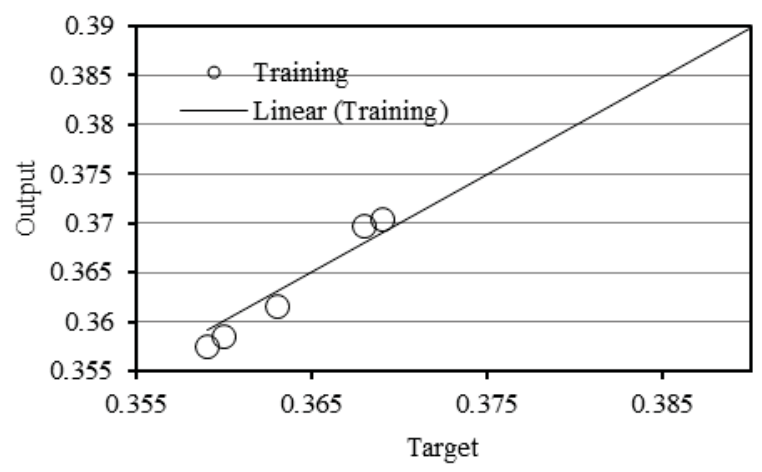

Fig. 4. Training regression line for long rod silicon rubber insulator.

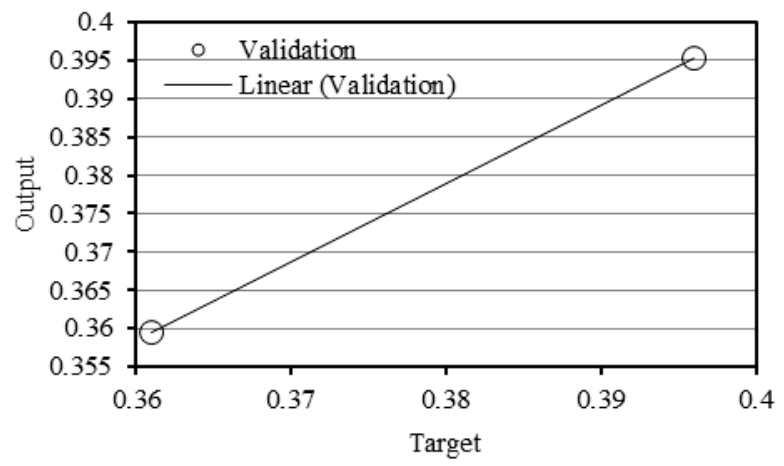

Fig.5. Validation regression line for long rod silicon rubber insulator.

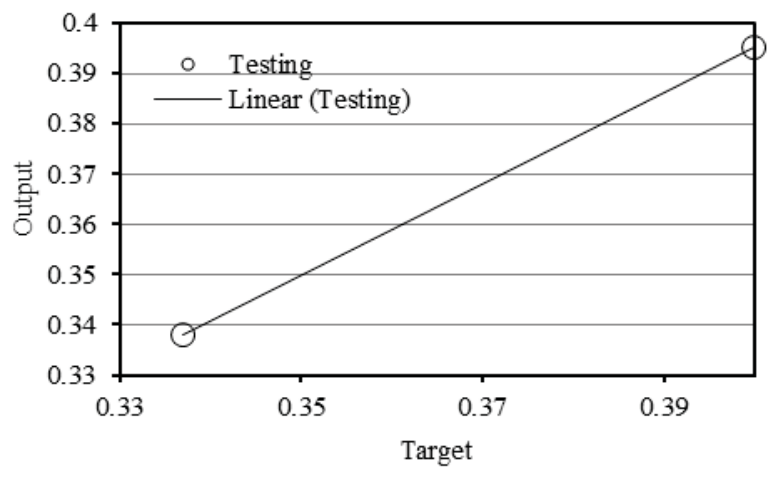

Fig. 6. Testing regression line for long rod silicon rubber insulator. 
Table II. ESDD comparison for long-rod silicon rubber insulator.

\begin{tabular}{|c|c|c|}
\hline $\begin{array}{c}\text { Insulators from } \\
\text { pin side }\end{array}$ & $\begin{array}{c}\text { Corrected ESDD } \\
\left(\mathrm{mg} / \mathrm{cm}^{2}\right)\end{array}$ & $\begin{array}{c}\text { Predicted ESDD } \\
\left(\mathrm{mg} / \mathrm{cm}^{2}\right)\end{array}$ \\
\hline$a(T)$ & 0.337 & 0.36221 \\
\hline$b(T)$ & 0.359 & 0.33712 \\
\hline$c(T)$ & 0.361 & 0.33903 \\
\hline$d(T)$ & 0.36 & 0.33732 \\
\hline$e(T)$ & 0.363 & 0.33755 \\
\hline$f(T)$ & 0.368 & 0.33781 \\
\hline$g(T)$ & 0.369 & 0.33794 \\
\hline$a(B)$ & 0.385 & 0.33715 \\
\hline$b(B)$ & 0.399 & 0.33714 \\
\hline$c(B)$ & 0.396 & 0.33713 \\
\hline$d(B)$ & 0.4 & 0.33713 \\
\hline$e(B)$ & 0.397 & 0.33712 \\
\hline$f(B)$ & 0.396 & 0.33712 \\
\hline$g(B)$ & 0.396 & 0.33712 \\
\hline
\end{tabular}

Table III. ESDD comparison for long-rod porcelain insulator.

\begin{tabular}{|c|c|c|}
\hline $\begin{array}{c}\text { Insulator } \\
\text { from pin side }\end{array}$ & $\begin{array}{c}\text { Corrected ESDD } \\
\left(\mathrm{mg} / \mathrm{cm}^{2}\right)\end{array}$ & $\begin{array}{c}\text { Predicted ESDD } \\
\left(\mathrm{mg} / \mathrm{cm}^{2}\right)\end{array}$ \\
\hline$a(T)$ & 0.257 & 0.22751 \\
\hline$b(T)$ & 0.238 & 0.24152 \\
\hline$c(T)$ & 0.215 & 0.22113 \\
\hline$d(T)$ & 0.234 & 0.21994 \\
\hline$e(T)$ & 0.219 & 0.22006 \\
\hline$f(T)$ & 0.232 & 0.22096 \\
\hline$g(T)$ & 0.232 & 0.2232 \\
\hline$h(T)$ & 0.236 & 0.22757 \\
\hline$I(T)$ & 0.232 & 0.23314 \\
\hline$j(T)$ & 0.236 & 0.23746 \\
\hline$k(T)$ & 0.234 & 0.23979 \\
\hline$l(T)$ & 0.235 & 0.24082 \\
\hline$m(T)$ & 0.235 & 0.24124 \\
\hline$n(T)$ & 0.235 & 0.24141 \\
\hline$o(T)$ & 0.235 & 0.24148 \\
\hline$a(B)$ & 0.245 & 0.24151 \\
\hline$b(B)$ & 0.269 & 0.24152 \\
\hline$c(B)$ & 0.282 & 0.24152 \\
\hline$d(B)$ & 0.263 & 0.24152 \\
\hline$e(B)$ & 0.259 & 0.24152 \\
\hline$f(B)$ & 0.279 & 0.24152 \\
\hline$g(B)$ & 0.281 & 0.24152 \\
\hline$h(B)$ & 0.281 & 0.24152 \\
\hline$I(B)$ & 0.282 & 0.24152 \\
\hline$j(B)$ & 0.253 & 0.24152 \\
\hline$k(B)$ & 0.281 & 0.24152 \\
\hline$l(B)$ & 0.281 & 0.24152 \\
\hline$m(B)$ & 0.282 & 0.24152 \\
\hline$n(B)$ & 0.281 & 0.24152 \\
\hline$o(B)$ & 0.285 & 0.24152 \\
\hline
\end{tabular}

\section{CONCLUSION}

Pollution level of long rod porcelain, and silicon rubber insulators have been measured. The pollution levels are found to be more than the IEEE and CIGRE standards which are identified as exceptionally high values. In case of long-rod porcelain insulator, the bottom surface has more pollution than the top surface. It means that the pollution on the bottom surface cannot be removed by the natural rain. The frequency of the rain in this tropical country is significantly high compared to other countries. The overall pollution level in the long-rod porcelain insulator is higher than the long-rod silicon rubber insulator. It is due to the pollution that adheres to the silicon rubber insulator surfaces for long time, and cannot be automatically removed by the rain. An artificial neural network has been applied to the measurement results and a good agreement has been achieved with the high determination of coefficients (more than 90\%). These high coefficients exhibit the strong correlation between the outputs and the targets.

\section{ACKNOWLEDGMENT}

The authors would like to acknowledge the support of the staff members who supplied the insulators to the laboratory of the Institut Teknologi Brunei.

\section{REFERENCES}

[1] E. Feilat, A. Al-Maqrashi, "ESDD- and DDDG- based Assessment of Insulator Pollution Levels in Oman", Proceedings of the $6^{\text {th }}$ IEEE GCC Conference and Exhibition 2011, UAE, pp. 593-596, February 2011.

[2] Mohammad Tariq Nasir, Muhammad Mysorewala, Lahouri Cheded, Bilal Siddiqui and Muhammad Sabih," Measurement Error Sensitivity Analysis for Detecting and Locating Leak in Pipeline using ANN and SVM", $11^{\text {th }}$ International Multi-Conference on Systems, Signals \& Devices (SSD), Barcelona, 2014.

[3] S. Nabavi, A. Gholami, A. Kazemi, M. Masoum,"Evaluation of Leakage Current Measurement for Site Pollution Severity Assessment", Leonardo Journal of Practices and Technologies, Vol. 10, pp. 39-54, 2004.

[4] M. A. Salam, Khaled Aamer, Ali Hamdan and Nasr Hamdan, "Study the Relationship between the Resistance and ESDD of a Contaminated Insulator-A Laboratory Approach", Proce. of the $7^{\text {th }}$ International Conference on Properties and Applications of Dielectric Materials, Nagoya, Japan, S15-5, pp. 1032-1034, June 1-5, 2003.

[5] Ahmad S. Ahmad, P. S. Ghosh, Syed Abdul Kader Aljunid and Hussein Ahmad, "Estimation of Salt Contamination Level on the High Voltage Insulators Surfaces During Rainy Season using Artificial Neural Network", Fifth International Conference on Power System Management and Control, Malaysia, pp. 303-308, 17-19 April 2002.

[6] IEC 60507 Publication, "Artificial Pollution Tests on High-Voltage Insulators to be Used on A.C. Systems," 1991.

[7] Muztoba Ahmad Khan, Saiful Huque and Azim Mohammad, "A Neural Network Model for Estimating Global Solar Radiation on Horizontal Surface", International Conference on Electrical Information and Communication Technology (EICT), Khulna, pp. 1-4, 13-15 February 2014.

[8] Wenxia Sima, Qing Yang, Xingliang Jiang, Jianlin Hu and Kunli Bai, "Outdoor Insulation Coordination with Artificial Neural Network", IEEE International Symposium on Electrical Insulation, Indianapolis, USA, pp. 316-319, 19-22 September 2004.

[9] Zhang Caiqing, Qi Ruonan and Qiu Zhiwen,"Comparing BP and RBF Neural Network for Forecasting the Resident Consumer Level by MATLAB", International Conference on Computer and Electrical Engineering, Phuket, pp. 169-172, 20-22 December 2008.

[10] M. A. Salam, SM Al-Alawi, AA Maqrashi, "Prediction of Equivalent Salt Deposit Density of Contaminated Glass Plates using Artificial Neural Networks", Journal of Electrostatics, Vol. 66, No. 9, pp. 526-530, 2008. 